\title{
Monocular occlusion impairs stereoscopic acuity, but total visual deprivation does not*
}

\author{
JOHN H. HERMAN, EDWARD S. TAUBER, and HOWARD P. ROFFWARG† \\ Department of Psychiatry, Montefiore Hospital and Medical Center, Bronx, New York 10467
}

\begin{abstract}
Wallach and Karsh (1963) reported that $24 \mathrm{~h}$ of monocular occlusion leads to a significant deterioration of stereoscopic depth estimates and attributed this phenomenon to "disuse." We designed an apparatus for testing stereoscopic accuracy which eliminated all cues to depth save binocular disparity. With it, we tested the relative effect of $8 \mathrm{~h}$ of monocular-as opposed to binocular-occlusion on subsequent stereoscopic performance. Monocular patching led to significant increases in mean standard deviation and in mean absolute error as compared to baseline testing. Binocular patching led to no such impairment. Thus, true disuse (such as occurs during binocular deprivation) did not impair stereopsis, whereas monocular occlusion, which may involve temporary misuse of the stereoscopic system, did.
\end{abstract}

Rapid modifications in estimates of depth based on binocular disparity have been demonstrated in human Ss by Wallach and Karsh (1963). Among various methods used to alter stereoscopic acuity, occlusion of vision to one eye was found to be effective. In this procedure Ss who showed accurate stereopsis on original testing of binocular depth perception wore a monocular patch for $24 \mathrm{~h}$. The patch was moved from eye to eye every 2 to $3 \mathrm{~h}$. The stereopsis testing apparatus used by Wallach and Karsh consisted of two pairs of vertical bars placed about $1 \mathrm{ft}$ behind an 8 -in. viewing window. The S's task was to adjust the depth between the two bars on the right, one of which was stationary, so as to match the preset depth of the two on the left. After $24 \mathrm{~h}$, which included the alternating monocular occlusion and a night of sleep, the Ss were retested. The standard deviation of the mean error increased $40 \%$, which for an $\mathrm{N}$ of 20 was highly significant.

Wallach and Karsh attributed the rapid deterioration of stereoscopic vision to the "disuse" of stereopsis during monocular occlusion. Total visual deprivation was not studied. They concluded that the "learning product on which the quantitative aspect of stereoscopic depth perception is based is not very durable . . . [p. 414]," and is constantly in need of fresn reacquisition. The term "disuse" was left unclarified in relation to specific mechanisms. However, Wallach and Karsh apparently assumed that monocular occlusion put the stereoscopic mechanism to rest.

*This research was supported by NIMH Grant 5-K05-MH-18739 and 2-R01-MF-13269 to Howard P. Roffwarg and by NIMH Training Grant 2-T01-MH-06418, which supported John $H$. Herman's postćoctoral fellowship.

tThe authors gratefully express their appreciation to Lloyd Kaufman of New York University for his help in designing the apparatus and writing this paper.
In terms of the hypothesis that "disuse" of retinal disparity results in a decline in stereoscopic acuity, we decided to determine whether or not binocular occlusion (total deprivation of visual input) had the same effect as, or perhaps an even greater effect than, monocular occlusion (partial deprivation of visual input) upon the accuracy of stereoscopic estimates.

\section{METHOD}

Six Ss, who demonstrated good stereopsis upon initial testing following $8 \mathrm{~h}$ of unobstructed vision (baseline condition), were chosen. Stereoscopic acuity was assessed by use of a modified Howard-Dolman procedure employing a technique that eliminated the possibility of size matching. The Ss' stereoscopic acuity was found to be stable as baseline accuracy was replicated on several occasions. These Ss also exhibited $20 / 20$ visual acuity, normal phoria, normal accommodation, and normal vergence/divergence.

The testing, which consisted of eight trials, was performed in a totally darkened room. Except when actually engaged in aligning the depth of two stimuli, the $S$ was instructed to keep his eyelids closed. The S's head was positioned on a chinrest while he adjusted one stimulus (the variable) until it appeared to be the same distance from him as a preset stimulus (the standard). The stimuli, mounted in sliding tracks, were each manipulated by a rope pulley. The pulley of the variable was placed in the S's hand.

The stimuli were two faintly illuminated translucent screens covered by diamond-shaped Aubert diaphragms. All sides of the diamonds were of equal length. The diaphragms, alterable for variation of the length of the sides of the diamong from 1/8 to $11 / 8$ in., were employed in order to eliminate size matching cues to distance. The luminance of each of the two diaphragms was approximately $0.2 \mathrm{fL}$, as measured by a Macbeth illuminometer. The stimuli were at eye level, and their centers were 7 in. on either side of the $S$ 's midline sagittal plane.

Following each of the eight trials, the $E$ reset one stimulus, using the left and the right alternately as the standard, at a distance of 6 to $8 \mathrm{ft}$ from the $S$. The variable was placed always at least $1 \mathrm{ft}$ away from the standard. After each trial, the lengths of the sides of the two diaphragms were altered so that they were always unequal. The E turned his back until the $S$ reported that he had finished setting the two stimuli at equal distances from himself.

A perfect stereoscopic depth estimate placed both stimuli in the same frontal parallel plane. Accordingly, after each trial, the 
distance in inches between the frontal parallel planes of the variable and the standard stimuli (the error) was measured. We report the standard deviation of each $S$ 's error scores as a measure of the variability of his stereoscopic performance independent of the magnitude of the error. The mean absolute error of each $S$ over eight trials is reported as an index of the relative accuracy of stereoacuity independent of the variability of the performance. Since the $S$ is his own control in each of three different conditions, reporting of mean absolute error provides an additional index of performance, that of an increase or decrease in accuracy as a function of condition independent of changes in standard deviation.

In contrast to the Howard-Dolman test (Howard, 1919), in which size matching is not eliminated as a cue to distance, the apparatus used in this study afforded a method of studying stereopsis that was uncontaminated by monocular cues (size matching is eliminated by the Aubert diaphragms; accommodation is an ineffective cue to distance past $6 \mathrm{ft}$ ). In a preliminary study with the identical apparatus, when the stimuli were identical in size, Ss were able to perform the task almost as well monocularly as binocularly. How. ever, when the sizes of the Aubert diaphragms were changed between trials, errors on monocular testing were frequently greater than $1 \mathrm{ft}$ whereas binocular performance remained unchanged. This demonstrates that, under certain conditions, depth estimates based on size matching approach the accuracy possible with stereopsis.

Following the baseline testing, the Ss were retested after each of two additional conditions according to a counterbalanced design: (1) all light occluded from both eyes by black patches for $8 \mathrm{~h}$; (2) all light occluded from one eye (the dominant eye) by a black patch for $8 \mathrm{~h}$. The eyes were patched at approximately $9 \mathrm{a} . \mathrm{m}$. and the Ss in both experimental conditions were tested after exactly $8 \mathrm{~h}$ and $10 \mathrm{~min}$. The additional $10 \mathrm{~min}$ allowed for dark adaptation by the monocularly patched Ss. During both conditions Ss were kept alert by an $E$, who was in constant attendance and encouraged conversation and responsiveness. At least 3 days, and typically 1 week, separated each of the three testing conditions.

\section{RESULTS}

In the baseline condition, i.e., after $8 \mathrm{~h}$ of unobstructed vision, the mean standard deviation on stereoscopic testing was 0.52 in. (see Fig. 1); following $8 \mathrm{~h}$ of monocular occlusion, the standard deviation was 1.47 in.; and following $8 \mathrm{~h}$ of binocular occlusion, the standard deviation was 0.77 in. At the mean stimulus distance of $7 \mathrm{ft}$, the visual angle of the Ss' stereoacuity was $38.00 \mathrm{sec}$ of arc on baseline

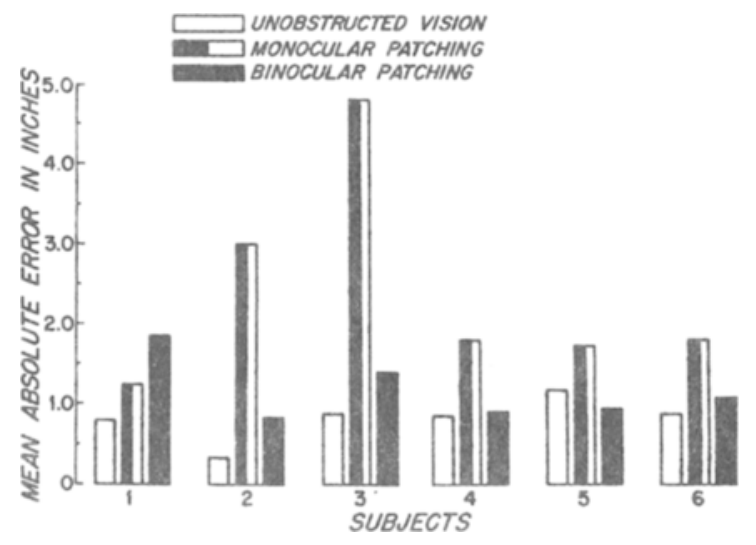

Fig. 1. Group mean standard deviations of the depth estimation errors for the total trials (all eight), first half of the trials (first four), and second half of the trials (last four) after each condition.

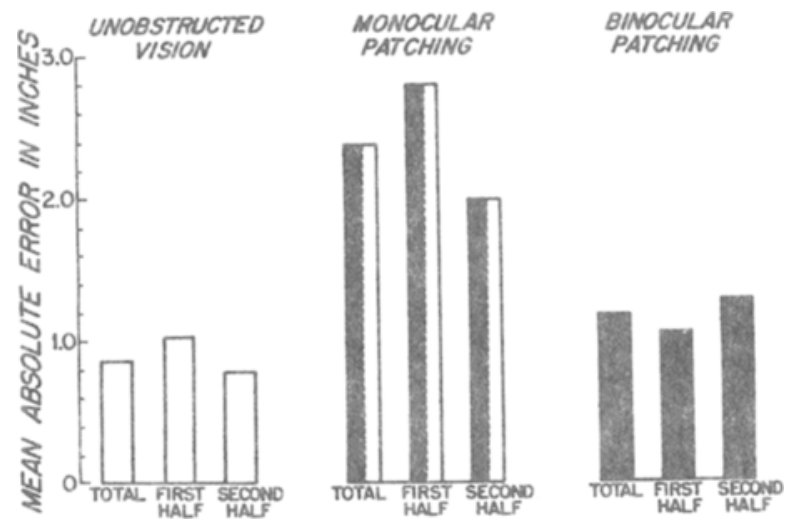

Fig. 2. Group mean absolute errors in Ss' stereoscoplc estimation of depth of the total trials (all eight), first half of the trials (first four), and second half of the trials (last four) after each condition. Total $=$ all eight trials.

testing, $107.4 \mathrm{sec}$ following monocular occlusion, and $56.3 \mathrm{sec}$ following binocular occlusion. The group mean absolute errors were $0.83 \quad(60 \mathrm{sec}), 2.4$ $(172.5 \mathrm{sec})$, and $1.1 \mathrm{in.}(78.9 \mathrm{sec})$, respectively (Fig. 2). No relation was observed between size of error, diaphragm size, or stimulus distance.

Since these were not predicted results, and because additional conditions had been tested, the Ss' error scores were analyzed via an overall Ss by Conditions ANOVA, one observation per cell. Statistical significance between treatments was assessed by Duncan's new multiple range test. The one-eyepatch condition was significantly different at the $<.01$ level from both the baseline and two-eyepatch conditions. The two-eyepatch condition was not significantly different from baseline. The effect size was striking in that monocular patching resulted in almost a tripling of the standard deviation (Fig. 1). Five of the six Ss showed a deterioration in stereoscopic performance after monocular occlusion in both standard deviation and mean absolute error relative to both baseline and binocular occlusion conditions (Fig. 3). One $\mathrm{S}$
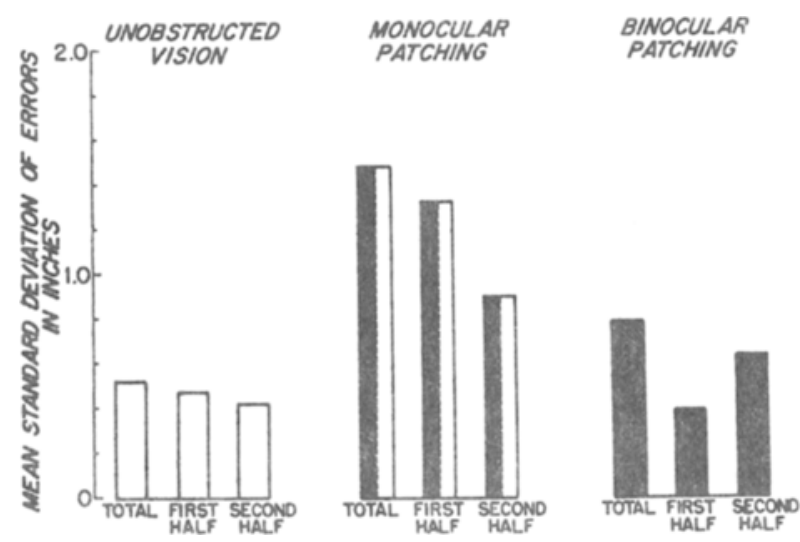

Fig. 3. Mean absolute errors of the six $S s$ in the baseline (unobstructed vision), monocular patching, and binocular patching conditions. Total $=$ all eight trials. 
(Fig. 3, S 1) showed the expected deterioration in monocular performance, but his stereopsis was impaired more by binocular than by monocular patching. There was no apparent explanation for his aberrant performance in this regard.

The standard deviations (Fig. 1) and the absolute error scores (Fig. 2) were calculated separately for the first four and the last four trials following each condition. The greatest improvement in performance between the first and the second halves of the trials occurred after one-eye patching. The group standard deviation of the first four trials was $1.31 \mathrm{in}$. and in the second four trials, $0.89 \mathrm{in.}$, a decrease of $32 \%$ (Fig. 1). The decrease in mean absolute error was proportional (Fig. 2).

\section{DISCUSSION}

This study confirms Wallach and Karsh's (1963) finding that binocular depth perception is a sensitive and reactive phenomenon easily impaired by a short period of monocular vision. However, our findings show that stereopsis is not compromised by simple "disuse," as a short period of restriction of all vision does not cause deterioration of binocular depth estimation. Rather, when vision is restricted to the monocular mode, aberrant or unbalanced utilization of the binocular depth apparatus temporarily impairs subsequent stereoscopic depth perception.

Berger and Scott (1970) have found greater stereoacuity $15 \mathrm{~min}$ after morning awakenings than prior to sleep onset. This accords with our finding that stereoscopic depth perception is not significantly impaired by an 8 -h period of disuse in the waking state. The six Ss described in our work were also tested for accuracy of stereoscopic acuity following a normal night's sleep, a night of REM deprivation, and a night of control awakenings. The data from these conditions will be the subject of another report.

Two possible mechanisms may be considered to underlie the impairment of stereopsis resulting from monocular occlusion: (1) interference with a key motor function, the visual oculomotor process that mediates the binocular alignment necessary for correct distance-disparity information; and (2) interference with a critical central sensory network through which disparity information from corresponding retinal points is properly integrated and utilized to yield accurate estimations of depth. One or both of these phenomena, each of which may develop specifically from the unbalanced condition of monocular occlusion and without connection to the other, would result not in simple "disuse" but in temporary misuse of the stereoscopic apparatus.

With reference to the postulated oculomotor imbalance, we suggest that the precise alignment of the two eyes, which is necessary for depth estimates based on retinal disparity, is impaired when only one eye is visually stimulated. Monocular patching may diminish the oculomotor balance necessary for precise phoria and vergence. As a result, imperfect ocular alignment would lead to faulty retinal disparity information during subsequent stereoscopic testing. When both eyes are deprived of visual input, the $S$ is presented with no visual-motor task and there is no interference with the fundamental organization of oculomotor coordination.

With reference to a central sensory integrating mechanism that mediates stereoscopic depth estimates, we hypothesize that such a system may be temporarily compromised in its information processing capacity during monocular occlusion. Under these conditions, dissimilar inputs arrive at the disparity detector cells of the cortex from the two eyes (patterned vision from the unpatched eye and blackness or dark discharge from the patched eye). This phenomenon results in a condition which virtually could be termed retinal rivalry. We postulate that under such conditions the "binocular depth cells" would neither be at rest nor be discharging in typical patterns, but instead would be in a state of physiological disorganization.

Following the period of monocular patching and the resulting impairment of either or both the motor and central sensory integrating processes necessary to stereopsis, a brief time lag seems to be required before the system can fully resume yielding veridical binocular depth information. Correction appears to take place rapidly after monocular occlusion. This is demonstrated by the improvement observed as the stereoscopic testing trials proceeded with the testing itself serving as practice. Sirce single-eye occlusion may affect either or both oculomotor coordination or a central sensory integrating mechanism, a further study will examine whether the rapid deterioration in accuracy of binocular depth estimates is a function of only one or both of these factors.

After patching the dominant eye of human Ss, Zubek and Bross (1972) have demonstrated an initial depression in the critical flicker frequency (CFF) of the nonoccluded eye, followed by an enhancement in performance. No such effects were observed in the occluded eye at corresponding times. The mean maximal depression in CFF followed 6-9 h of patching corresponding to our experiment's 8-h test period. Their study and ours, taken together, suggest that monocular occlusion uniquely produces multimodal performance decrements in central integrating mechanisms of the binocular visual system.

It is of interest that the results of this study of performance in stereoscopic depth estimates are paralleled by the long-term effects of monocular patching, as opposed to binocular patching, in very young animals (Hubel \& Wiesel, 1962; Wiesel \& Hubel, 1965; Guillery, 1972). These studies show that deprivation of monocular input seriously affects the 
development and responsiveness of cells in the lateral geniculate nucleus as well as in the visual cortex. Binocular occlusion leads to little, if any, structural or functional impairment.

The deterioration of stereoscopic acuity found under the conditions employed in this study raises additional questions. Why is the magnitude of the effect after $8 \mathrm{~h}$ in this study greater than that observed after $24 \mathrm{~h}$ by Wallach and Karsh? A possible explanation is that elimination of accommodation and size matching cues to distance with our apparatus allowed an assessment of pure stereopsis and, therefore, a truer estimate of the decline in this function. Another difference in the two studies is that Wallach and Karsh may have modulated the extent of impairment in their study by switching the patch from one eye to the other every 2 or $3 \mathrm{~h}$, whereas we left the S's dominant eye patched the entire time.

Still to be investigated are the issues concerning rates of falloff and recovery of function. For example, how rapid is the worsening of stereopsis after occlusion of vision to one eye? Does the effect observed in this experiment require $8 \mathrm{~h}$ to develop or is an asymptote reached in performance error after only a brief period? Likewise, would a greater decrement result from a lengthened period of monocular patching, or would a significant decrement develop after a longer period of binocular deprivation? Along these lines, it would be of interest to explore whether impairment of depth estimating capacity in children results from the long periods of monocular patching employed after corrective ophthalmological procedures. Although we have no data that bear directly on the latter question, as indicated, we have noted a rapid improvement in performance during the stereoscopic testing procedure itself.

\section{REFERENCES}

Berger, R. J. \& ScotT, T. D. Increased accuracy of binocular depth perception at the end of REM periods. Paper presented at the meeting of the Association for the Psychophysiological Study of Sleep, Santa Fe, New Mexico, March 1970.

GuILleRY, R. W. Binocular competition in the control of geniculate cell growth. Journal of Comparative Neurology, 1972, 144, 117-130.

Howard, H. J. A test for the judgment of distance. American Journal of Ophthalmology, 1919, 2, 656-675.

Hubel, D. H., \& Wiesel, T. N. Receptive fields, binocular interaction and functional architecture in the cat's visual cortex. Journal of Physiology, 1962, 160, 106-154.

Wallach, J., \& KarSH, E. B. Why the modification of stereoscopic depth perception is so rapid. American Journal of Psychology, 1963, 76, 429-435.

Wiesel, T. N., \& HuBel, D. H. Comparison of the effects of unilateral and bilateral eye closure on cortical unit responses in kittens. Journal of Neurophysiology, 1965, 28, 1029-1040.

ZubEK, J. P. \& Bross, M. Depression and later enhancement of the critical flicker frequency during prolonged monocular deprivation. Science, 1972, 176, 1045-1047.

(Received for publication June 18, 1973; revision received March $12,1974$. 